and dimensions of the seams offered a more favourable set of circumstances for commercial development. Nevertheless, in those cases considerable pioneer work had to be undertaken before any feasible projects emerged.

The book is well produced. It is clearly printed and well illustrated. It would indeed bo a boon to the scientific world if all projects of equal merit could be so well reported.

R. J. SARJANT

\section{MATHEMATICS FOR PHYSICISTS}

Mathematical Mathods of Physics

By Dr. Jon Mathews and Dr. R. L. Walker. Pp. $x+$ 475. (New York and Amsterdam: W. A. Benjamin, Inc., 1964.) 13.75 dollars.

$\mathrm{P}$

ART of the equipment of a moderm physicist is a sound knowledge of a wide variety of techniques and theories in pure mathematics. If his interest is in theoretical physics he may have to be thoroughly expert in tho theory of functions of several complex variables, Lie groups and functional analysis; even if his own work is primarily in the experimental field he still needs to have a 'reading' knowledge of a wide variety of mathematical methods if only to be able to read the theoretical papers relating to the subject of his own researches. This requirement places a heavy responsibility on teachers of mathematics to provide courses which are at the same timo interesting, comprehensible and valuable to undergraduate and graduate students of physics. To anyone charged with such a task the present book will bo of interest, since it shows one possible way of proceeding in the sense that within the space of 458 pages (sixteon chapters and an appendix) it attempts to present an account of the major branches of mathematics which are of interest to physicists. Since corresponding to each of the chapters but one there is at least one well-known monograph of roughly the same size as the present volume, it is obvious that the various topics discussed cannot bo treated in a completely satisfactory fashion.

Tho book consists of the following chapters: (1) ordinary differential equations - a briof review but including a fairly full account of the Wentzel-Brillouin-Kramers method; (2) infinite series; (3) evaluation of integrals ; (4) integral transforms -with an account of Fourier series and the Dirac delta function; (5) further applications of the theory of functions of a complex variable (conformal transformations and dispersion relations); (6) voctors and matrixes including (in 25 pages !) the theory of linear spaces and spaces of infinite dimensionality; (7) special functions (Legendro, Bessel, Hermite, Laguerre, hypergeometric, confluent hypergeometric and elliptic); (8) partial differential equations-mainly characteristics, separation of variables, use of integral transforms, Wiener-Hopf method; (9) eigenfunctions, eigenvalues and Green's functions; (10) perturbation theory; (11) integral equations-with applications to dispersion theory; (12) calculus of variations - and its connexion with eigenvalue problems; (13) numerical methods (interpolation, numerical integration, numerical solution of differential equation, the summation of series; (14) statistics and probability; (15) tensor analysis and differential geometry, made up of sections on cartesian tensors in three-dimensional space, curves in three-dimensional space (the FrenetSerret formulæ) and general tensor analysis; (16) groups and group representations consisting of sections on groups, group representations and characters, infinite groups. Finally there is an appendix of some proporties of functions of a complex variable-provided as a review for most readers but as a study guide for readers unfamiliar with tho subject already.

Both Dr. Mathows and Dr. Walker are physicists, and they have constructed the book from notes which they used in giving a course in mathematical mothods in physies to first-year physies graduate students and senior physies undergraduates at the Californian Institute of Technology. This is reflected in the finished product. The choice of mathematical topies is dictated by their established usefulness in physics, the material is illustrated by examples chosen from mechanics, electricity and magnetism, ard wavo mechanics, and the level of rigour is that at present accepted in theoretical physics.

In a volume of this size covering so very many topics it is to be expected that most of the topics are treated in much the same way as they would be in an encyclopxdia such as the Encyclopodia Britannica or the Pergamon Press Encyclopoedic Dictionary of Physics; but it should be emphasized that they are first-rate articles. 'The basic definitions and points of theory are clearly stated, the relevance to physics brought out and illustrative examples provided; there are also problems provided by means of which the reader can test his comprehension of the text (but no answers are provided).

The authors are to be complimented in having covered so much material so well in a limited space and par. ticularly in having shown (as so many books in this area fail to do) that there are algebraic methods valuable in physics as well as analytical ones. The nature of undergraduate and postgraduate courses in Britain is so different from the scheme set out in this book that it is unlikely to be of much value to students attending such courses - except as a valuable reference book. It should, however, be studied by university teachers conducting such courses and by scientists and students who have to study the subject on their own, for it will provido them with a clear statement of the fundamentals of most of the topics they are likely to be interested in and guidance towards a deeper study.

I. N. SNEDDON

\section{MATHEMATICS OF RUSSIAN TECHNOLOGY}

\section{A Course of Higher Mathematics}

By V. I. Smirnov. Vol. 1: Elementary Calculus. Pp. xiii +546 . Vol. 2: Advanced Calculus. Pp. xiv +632 . Translated by D. E. Brown. Translation edited by I. N. Sneddon. (International Series of Monographs in Pure and Applied Mathematics, Vols. 57 and 58.) (London and New York: Pergamon Press, 1964.) 90s. net each volume.

$T$ HESE are the first two of a set of five volumes, which cover the topics of mathematical analysis likely to be of use to the engineer and physicist, from the elements of calculus up to partial differential equations, integral equations, set theory, Stieltjes and Lebesgue integrals, normed spaces, and Hilbert space.

The book first appeared in 1924, a collaboration between Tamarkin and Smirnov; after the second edition, Smirnov's name appeared alone. The present version is a translation from the sixteenth Russian edition. Smirnov, though perhaps less well known in the West than Tamarkin, has had a distinguished career, with researches of importance in both pure and applied mathematics, as well as a wide teaching experience. This broad outlook prompted him to construct a comprehensive course of higher mathematics which should have an immediate appeal to the top-class engineer and physicist, without ignoring the demand of the pure mathematician for rigorous exposition.

Volumes 1 and 2 deal with the classical results of the infinitesimal calculus, including vector analysis, differential geometry, Fourier series, and the simpler partial differential equations of mathematical physics. In such a well-gleaned field, no great originality is to be expected, and the main interest lies in seeing how the author has coped with the two-fold demand for physical applicability and for mathematical rigour. The means adopted is the division of the text into two type-founts; in the large 